\title{
Application of Case Study Methodology in the Exploration of Inclusion in Education
}

\author{
Prativa Shrestha and Prakash C. Bhattarai ${ }^{1}$ \\ Kathmandu University, Nepal
}

\begin{abstract}
The purpose of writing this article is to demonstrate that the case study methodology can be very useful in the exploration of inclusion in education. As evidence, the authors have presented their experiences of using a case study methodology to study inclusive education with a particular focus on girls with visual disability and their school context. Going beyond an interpretation aligned with ecological system theory and theory of dysontogenesis, the methodology was able to capture the essence of the participants' experiences, exploring the different layers of environment and how the layered aspects influenced the school experiences of girls with visual disabilities and their integration or segregation in schools. In the meaning-making process, the case study was able to unpack the complexity of school and classroom practices and their connections amongst diverse layers at the levels of the teacher, the pupil, the classroom, the school, and its context.
\end{abstract}

KEYWORDS: inclusive education, inclusion in Nepal, research in inclusion, case study, visually impaired girls.

Inclusion education is taken as an approach ensuring the right to education of all children including the poor, marginalized, and disadvantaged (Regmi, 2017). According to Mcmanis (2017), inclusive education takes place when all children irrespective of any abilities or disabilities such as emotional, physical, or neurological that they may possess are included in age-appropriate general education classrooms in their local schools to obtain quality education to meet the achievement of their core curriculum requirement. Considering the importance of the issue, several studies in the area have been carried out following diverse methods. This article provides some major advantages of the case study methodology in the exploration of inclusive education. To illustrate these advantages, an example of exploring visual disabilities by using the case studies is presented. The argument is that a case study can be beneficial to unpack the complexity of inclusive education. The article begins with the discussion of inclusive education in Nepal, where this study has been carried out. The following section consists of the standpoint and description of the case study research that the researchers undertook. Additionally, the benefits of using the case study in this disability research have been discussed. The final section of the article discusses the issues and challenges that the authors faced and the corrective measures which could be applied in carrying out this study using the case study methodology.

1 Corresponding Author: An Associate Professor and Head- Department of Development Education,

Kathmandu University-School of Education, Nepal. E-Mail: prakash@kusoed.edu.np 


\section{Inclusive Education in the Context of Study Area}

The development of inclusive education in Nepal was initially influenced by the disability movement. However, this does not mean that educational reform movements did not have a significant role in the origination and development of inclusive education. In this context, two specific movements namely a) school improvement, and b) special needs education have supported the development of inclusive education in Nepal. The wave of inclusive education in the form of special education in Nepal commenced in 1967 A.D. with the operation of an integrated education program for visually impaired students in the Laboratory School, Kathmandu. This momentum got its expansion through the implementation of the National Education System Plan (1971-1976). The formation of the Special Education Council in 1973 was one of the outcomes of this plan. However, it could not develop and expand as expected because of several reasons such as less understanding of the importance of special education by the government and social sector, lack of human resources, lack of physical and instructional facilities and inadequate financial resources, etc. ( $\mathrm{T}$ U, 2015). Subsequently, the Education Act 1971 provided provision of an educational facility to Special Education Need (SEN) children with visual, hearing, intellectual, and mental disabilities. Moreover, under the National Education System Plan (NESP), the Special Education Council (SEC), an authorized body for the educational arrangement of SEN children was established in 1973. This was a landmark in the history of SEN children's education in Nepal. Then the government took the responsibility of SEN children's educational access like non-disabled children (Thapa, 2012) and there has been development in the policies thereafter.

The Department of Education (2007), now called the Centre for Education and Human Resource Development (CEHRD) under the Ministry of Education, Science and Technology (MoEST) has identified its target groups of inclusive education policies of which disabled children is one of them among others (ethnic and linguistic groups, street children, child laborers, children affected by conflict and trafficking, orphans, children living in poverty, children with HIV/AIDS and Leprosy, Kamaiya or bonded labor children, children studying in Madrasa, Gumba or monasteries, children from linguistic minority, refugee children, and remote geographical location. However, the authors have limited the present article to inclusive education in terms of disability and gender with a particular focus on visual disability.

Several initiatives have been launched to impart integrated and inclusive education for children with disabilities by the MoEST, development organizations, charity organizations as well as religious institutions. The Government of Nepal has adopted many policies for increasing the participation rate of children with disabilities in education. The Constitution of Nepal, 2015 in its Clause (31) ensures provision for free higher education for children with disabilities, economically poor, visually impaired, and also for those who are with hearing impairment (Government of Nepal [GON], 2015)

Inclusive Education Policy, 2017 has also reiterated need-based course books for visually impaired students including textbooks in Braille to be provided. Further, the policy has made provision for setting up inclusive education minimum enabling conditions at schools and functional assessments to be undertaken while going to admission in schools for children with visual disabilities. Education for children with disabilities took a turn from welfare-based approach (Disabled Persons Welfare Act, 1982) to the right-based approach (Nepal Law Commission [NLC], 2017). However, the policy has not been serving its intent to the full extent with regards to disabilities in general, including visual disabilities. The recently formulated National Education Policy 2019 (MOEST, 2019) has made provisions for opportunities to be imparted to people with disabilities for inclusive and special education taking into consideration

the lifelong learning skills and textbooks for curriculum. The Act related to Compulsory and Free 
Education 2018 (NLC, 2018), states that a citizen with a disability shall also have the right to get education using special education. Despite the various provisions of inclusive education at the policy level, many children in Nepal are unsupported from educational provisions of school, and many children leave school before completing primary education among those who come to attend also. In addition, many children suffer from positive learning outcomes and class repetition has been an issue of concern (Regmi, 2017).

According to Central Beaureau of Statistics (CBS)(2012), physical disability including blindness and/or low vision covered more than half of the total number of disabilities. Disability in rural residents was more prevalent (2.1 percent) compared to disability in their urban areas (1.2 percent). Fatima et al. (2013) identified that visual disability can induce isolation, can build obstacles to the attainment of degrees leading to unemployment. However, visual disability is not an issue in itself but that illiteracy and poor self-esteem make life difficult for individuals with visual impairments. Therefore, the actualization of proper inclusive education requires appropriate pedagogies as well as positive social interactions responding to the educational needs of diverse students (Boyle and Anderson, 2020). Meanwhile, this inability of actualizing inclusive education can cause discrimination among the students. Confirming this finding, Morris (2014) has revealed in her study that the willingness of visually disabled students to enter teaching and learning and becoming independent in potential educational and job environments will serve as a challenge. This finding is also confirmed by Lamichhane (2012) in his mixed-method study depicting people with visual and hearing impairments and challenges encountered by insufficient services in classrooms, whereas girls with physical disabilities were primarily met with difficulties created by physical obstacles, such as inaccessible housing and lack of safe, open roads.

Besides, the obstacles faced by girls, in general, were financial, as a result of parental poverty, and barriers as a result of parental perceptions, social stigma, and lack of understanding. Additionally, Thapa (2012) in her phenomenological research found that teachers are facing difficulties in the testing and examination of visually impaired learners. Teachers are not in a position to review the students' response sheets in the test. Teachers encountered challenges in integrating visually disabled learners with vision students. Research studies (e.g. Regmi, 2017) reveal the practice of inclusive education in the form of fitting a needy child in the education framework of Nepal. For the author, this is a misconception of inclusive education and this cannot be taken as an inclusion; indeed, it is rather assimilation.

In this context, the authors identified that the real phenomena of the visually impaired girls in different circumstances had not been explored yet, and the study was important to identify whether the visually impaired girls feel integrated or segregated in their school. Additionally, while working in the field of gender and inclusion the first author encountered many issues related to the topics that were unheard and unspoken of. In exploring the phenomena, the authors identified that case study methodology can be one effective way of exploration to understand especially when such holistic phenomena are involved if inclusive education has been practiced. The following section discusses the standpoint of the authors with the methodology of case study.

\section{Our Standpoint in Case Study Methodology}

The current study was conducted among girls with visual disability employing a case study methodology. The authors followed Merriam and Stake since they are seminal authors. They are the methodologists who provide procedures to follow while conducting case study research. Yin's perspective on case study was also critically considered (Bhattarai \& Maharjan, 2016). The authors 
had two specific considerations while carrying out the study. Firstly, a case study is an approach which involves studying individual cases in their real-life context for a long period, which in our study was cases as a single unit of analysis. Five girls with visual disabilities were considered as cases for analysis. Secondly, similar to Yin (2018), Each case study was considered as a contemporary phenomenon inside its real-life setting, particularly when the boundaries between a phenomenon and its context are unclear and the researcher has limited influence over the phenomenon and context. This was depicted in this study by understanding the experiences of girls with a visual disability under different circumstances through an emic perspective.

The researchers who are the authors themselves were guided by the characteristics of qualitative research such as holistic, empirical, interpretive, and emphatic (Stake, 2006). The term 'holistic' in this context refers to the totality of the contexts and the phenomenon under the study. To maintain holistic characteristics, this study captured the experiences of girls with visual disabilities from multiple layers such as their experiences in family, peers, teachers, socioeconomic status including the policies that support (or do not support) in imparting inclusive education. For the authors, empirical means that researchers base the study on their observations in the field. This study was empirical since it was field based. Interpretive means that researchers rely on intuition and consider research as primarily a researcher-subject interaction, which is consistent with constructivist epistemology. The interpretive paradigm was adopted to make the study interpretive with multiple realities. Lastly, empathic means that from an emic standpoint, researchers represent the respondents' vicarious experiences which was also reflected in this study through the worldview of the visually impaired girls studied to explain the particular values, beliefs or practices. Merriam (2009) also believes that the case under a study is delimited depending upon their scope, function and/or given boundaries.

The cases in the study were studied in the form of culture, society, community, organization, or phenomenon, such as beliefs, practices, or interactions (Harrison et al., 2017). The researchers developed guiding themes for interviews accordingly which would capture information on the participants' cultural and economic background, their interaction with family, teachers, and friends, the home school relation among others. We refer to research as cross-case when the emphasis moves from the individual case to more than one case. The distinction between a case study and a cross-case study is due to the matter of degree. The fewer cases there are and the more intensively they are researched, the more merit for case study (Gerring, 2007). The case study was limited to five individual participants only with intensive interviews multiple times. Observations were also made onsite to gather deeper insights. Observations regarding the school infrastructure, inside classroom while teacher was teaching, the interaction of participants with their peers, participant's room in the hostel and the gestures while interviewing them were noted which were considered responses to generate study findings.

The purpose of the case study varies, depending on its type. Out of multiple and single case study designs, the undertaken research was a multiple case study since five different girls were considered for the study. A single-case study emphasizes a single case only, whereas multiple-case studies include two or more cases in the same study. This article, therefore, highlights our research on inclusive education with a focus on girls with a visual disability with some methodological aspects of qualitative case study designs and its usefulness for research on inclusive education. The author's justification for applying the case study method lied in the fact that the study research questions were mainly descriptive as it aimed to produce a firsthand understanding of girls with visual disabilities and events of their experiences.

The study had some delimitations. The study was undertaken within the Kathmandu Valley in the integrated schools only. It was limited to the girls with visual impairment who were studying 
in the given schools. Only the issues of education related to those girls were focused under the study.

\section{The Research of Inclusive Education by Using Case Study}

Inclusive education was delimited to the experience of school-girls with a visual impairment in their school experiences. The research had two research questions: (a) What are the layers of the environment influencing the school experiences of visually impaired girl students? and (b) How do the layers of environment influence in integrating or segregating the school experiences of girls with visual disability. This research was based on a case study methodology that explored the aspects of different layers of environment and how those layered aspects influenced the school experiences of girls with visual disability for integration or segregation. The theoretical lenses used in the study were Bronfrenbrener's (1979) Ecological Systems Theory and Vygotsky's Theory of Dysontogenesis (1998). The ecological system theory explains the different layers of the environment affecting the development of an individual. Since our research concern was also to explore the layers of concerns affecting the school experiences of girls with a visual disability, the findings were theorized with ecological system theory. Likewise, the researchers were also guided after the identification of findings by Vygotsky's theory of dysontogenesis which is influenced by the social constructionist model on disability. For Vygotsky, disabilities are not to be considered only in the form of physical impairment but its consequences to social environment and dialectical interaction. Thus, the social environment is crucial in the upbringing of children with disabilities (Vygotsky, 1998). Limited mobility due to the exclusionary environment excludes girls with visual disabilities from many education opportunities. Both these theories were aligned after the study findings were generated during the meaning-making process.

The study was undertaken in two integrated schools with five visually impaired girl students as the main participants. The participants were selected purposely. On the way to selecting participants, the authors explained study objectives to get support in selecting the girls who would contribute to meet the purpose at their best. The schools were in Kathmandu and Lalitpur districts. Both were integrated schools. One of them was an institutional school and the other one was a community school. Both schools had visually impaired students along with sighted students studying together in a class. Both schools had a separate visually impaired 'Resource Centre', integrated into schools. English was strictly adopted as a medium of instruction in the institutional school whereas all subjects except English were taught in Nepali in the community school. Community schools were considered inefficient in comparison to institutional schools in their performance. The aspirations of the participants from the institutional school were high. They were more ambitious regarding their plans than the community school participants. Mathema (2007) has also agreed that the growing disillusionment with the public-school system is a result of its continued poor results. The participants were studying in grades 7 to 10 . All the girls come from various districts from the eastern to the western part of Nepal namely (a) Kaski, (b)Syangja, (c) Okhaldhunga, (d) Morang, and (e) Dailekh representing diversity in terms of geography too. They all came to Kathmandu to seek education in school due to a lack of proper schools for them in their districts. All of them except one participant were residing in a hostel.

In addition to the main participants, the teachers and parents of the visually impaired girls have been interviewed as secondary participants to gather insights on the background information. For the selection of cases, first of all, the researchers identified the layers that support and provide a conducive environment for girls with visual disabilities. Of course, researchers were conscious to keep in mind that girls with disabilities who come from more educated, aware, and well-off 
families will have higher chances to receive quality education than the girls who do not have the same resources. Field work started with an informal discussion with the girls. The interview was taken with one student in a day. A minimum of three rounds of interviews with each participant was taken. Two students were interviewed four times as they did not open up with the authors initially. In addition, classroom observation was also done to see how the visually impaired students participated in the classroom and how they interacted with peers. An observation of the physical environment of the school was done to assess whether it was disability-friendly or not. Interviews and observations were common with all three case study methodologists i.e., Yin, Merriam, and Stake for gathering information (Yazan, 2015). The interviews were based on some newly evolved issues that necessarily helped in structuring the observation, interviews, and document review (Stake, 2006). The information was analyzed based on the interviews, observations, and reviews of past research, policies, and legislation as well as theoretical connections.

The study was carried out with the six-stages of the case study protocol as the researchers wanted to enrich their study by integrating additional guidelines from the wider methodological literature (Baskarada, 2014). The six stages followed were: (a) Plan (b) Design (c) Prepare (d) Collect (e) Analyse (f) Share. Research questions for the case studies were identified in the planning stage. While confirming the case study as the key methodology, its strengths and limitations were also discussed connecting it with the research questions. While planning for employing the case study as a research methodology, the author ensured that both the research questions and the case study method support each other (Baskarada, 2014). The case study method followed was of more of an explanatory nature since it tried to dig into a "how" research question. Mainly three main criteria as recommended by Stake (2006) were followed in selecting our cases i.e., (a) Was the case relevant to the issue? (b) Did the cases provide diversity across contexts? (c) Did the cases provide good opportunities to learn about complexity and contexts?

The researchers identified a single case as the unit of analysis in the design stage. It helped in generating theory/propositions and identifying difficulties underpinning the expected study for defining the case study design. The researchers followed multiple case study designs, as it studied five cases of visually impaired girls from different backgrounds. During the research design, researchers were guided by Merriam. The five steps of Merriam include a literature review, constructing a theoretical framework, identifying a research problem, crafting and sharpening research questions, and selecting the sample i.e., purposive selection (Merriam, 2009). The study followed four defining characteristics for designing qualitative case studies which are holistic, empirical, interpretive, and emphatic as pointed out by Stake (2006). To make the study empirical, it is based not only on the discussions, in-depth interviews but also on class observations and school infrastructures.

The study preparation stage focused on developing the researchers' skills as a case study researcher, developing a case study protocol, contextualizing it, and gaining any relevant approvals (Baskarada, 2014). This preparation also aimed to identify any relevant issues in the case study design and endeavor to address any such issues before starting the information collection stage. Once the guiding questions were developed, it was contextualized with visually impaired students of 12th grade from another school to determine relevance and try to assess whether the questions facilitated the research details before conducting the in-depth interview and discussions with visually impaired girls finally.

The collection stage involved following the case study protocol, using multiple sources of evidence, creating a case study database, and maintaining a chain of evidence. In-depth interviews and discussions were our main source of information. Besides, there was equal reviews of empirical studies from various journals from Jstor, Sage, Journal of Literary and Cultural Disability Studies, 
International journal of inclusive education, dissertations among others, and did observation in the classrooms, physical features of the school, the interaction of visually impaired students with their peers. The analysis stage was based on theoretical ideas and other tactics, considered and used analytic tools, investigated competing explanations, and exhibited information (facts) separately from interpretations (Baskarada, 2014). Indeed, qualitative analysis was the most difficult and the least codified part of the whole case study process. The research strategy was guided by Merriam (2009) in which analysis is the stage of sense-making. The process of sense-making includes consolidating, reducing, and interpreting what people have said, and what researchers had seen and read - it was the process of meaning-making (Yazan, 2015). The raw information gathered was first transcribed and written down in our notes by organizing it into categories based on themes and depending on the sources of information. Secondly, a generalized understanding of the information and its overall significance was represented, such as how the overall depth, credibility, and use of the information was seen. Finally, as described by Baskarada (2014), the sharing stage concentrated on defining the audience, combining textual and visual materials, providing enough evidence for a reader to reach our findings, and reviewing and re-writing till done effectively.

The study followed university guidelines of ethics. Informed consent was taken in which the girls agreed for their participation without any duress before the research got underway. All necessary steps to ensure that all girls in the research understood the process in which they were to be engaged, including why their participation was necessary. The girls were treated with respect and they were assured that they could withdraw from the research if they felt so. Recognition of the right of the participant to withdraw from the research for any or no reason and at any time was practiced. The confidentiality and anonymous treatment of the participant were considered duly. The pseudonyms for all the girls and schools were used to protect the identities and confidentiality of the girls.

Best efforts were made to make the study credible and maintain trustworthiness. Multiple consultations were made as pointed out by Baxter and Jack (2008). Verifying of the information was done with different data sources of information by examining evidence from the sources and using it to build coherent justification for themes as spelled out by Creswell (2007). The information gathered was rigorous and the descriptions were detailed. There were multiple layers of information collection. As suggested by Rashid et al. (2019), the findings from other studies were also compared with the study. In addition, the authors looked into many aspects of the research such as accuracy of transcription, the relationship between the research question and the information, the rigor of meaning-making and analysis, etc (Haines, 2017).

The study identified five layers of environment namely: a layer of direct influence, a layer of indirect influence, a layer of policy and legislation, a layer of socioeconomic status, and a layer created by school stratification that had influenced the school experiences of girls with visual disability. Girls in this study had similar school experiences, sometimes feeling integrated and sometimes feeling segregated. Girls' progress and schooling experiences had been influenced by supportive relations between family, peers, and teachers in providing support and learning, by professional and policy legislations i.e., the laws relating to people with disability for imparting inclusive education, and by the school stratification and the socio-economic status of their family.

The approach of integrated education had observable pros and cons for the visually impaired girls. By studying together with sighted students, they were provided with the opportunity to compete with one another thereby increasing their social harmony which one sense gave them yet more opportunities to become competitive. However, there was a blanket approach provided by the school to all students. They had to accommodate themselves in a "one size fits all" approach and this had been a barrier for the visually impaired girls. They are thus socio-culturally challenged 
more than their visual impairment. However, the lack of adequate facilities did not lower their determination to pursue their education and desire to become self-reliant in the future. They have been able to cope despite feeling segregated but are very positive in their outlook.

As a part of the implication of the study, the school authority including teachers and parents can be provided with an orientation for developing disability-friendly policies. In addition, the policies so far have been formulated only through the eyes of primary disability. If the focus of these policies is shifted towards secondary disability, the visually impaired student will not form another kind of socio-cultural disability.

\section{Benefits/ Strengths of Using Case Study}

Using a case study methodology was very useful for the present study as case study research is richly descriptive, can dig in-depth and varied sources of information. The researchers were able to identify the enablers and barriers affecting the school experiences of girls with visual disabilities. The information was not only dug out from primary participants but also secondary participants. Quotations from key participants, anecdotes, interviewed writing, and other literary methods to construct mental representations that brought to life the ambiguity of the multiple factors involved in the phenomenon under study by visually impaired girls were used (Hancock \& Algozzine, 2006). Through the case study methodology, the authors were able to capture the holistic situation of the research participants regarding the different circumstances which caused them to feel included and sometimes excluded in their schools' experiences, for example, the researchers were able to dig into the concerns of the services for visually impaired girls to meet their unique educational and learning needs, particularly their needs for instruction in reading, writing, and composition.

One of the distinct features that differentiate the case study methodology from other methods is that both single and multi-case studies focus on particularization more than the generalization of the findings. Stake (2006) also believes that the power of the case study in the research is its attention to the local situation, not in how it represents other cases in general. Through the in-depth information generated from the case study methodology, the research findings were able to link it with both the Ecological Systems Theory and Vygotsky's theory of Dysontogenesis. Not only were the findings limited to the proposition of the theory, but they also came up with another layer of influence in the finding which was beyond what was stated by the theory. It was a very interesting finding of how the two types of schools were creating stratification among the participant's learning experience which made their experiences segregated. Additionally, in the case of studies, diverse information sources are tapped to chart the unique case in its natural environment as evident in identifying different layers of environment for acting as enablers or barriers in-school experiences of girls with visual disabilities in the research. This is because the case study delves into the issues in detail by probing into events that happen in a natural setting. The case study methodology was also equally flexible. After the selection of a topic, the decision on the boundaries of the topic depending on the extent of the research topic was made. When cases were selected carefully, the design of a study could incorporate a diversity of contexts with interesting findings such as the stratification created by schools as discussed above which was beyond what theory had in the proposition.

The case study approach appeared to be relevant in researching inclusion. It contributed to going in-depth on the issue, event, or phenomenon of interest about the given time and context (Mukhopadhya et al., 2012). It enabled the researchers to dig into important topics that could not be easily covered by other methods. The researcher's justification for applying the case study method lied in the fact that the questions were mainly descriptive as the research questions aimed 
to produce a first-hand understanding of girls with visual disabilities and events in their lives helped which helped to examine cases from historical to present form and bring out the similarities and dissimilarities among the cases.

\section{Lessons Learnt in Using Case Study in Inclusive Education}

A case study is an intensive study of specific phenomena to simplify the understanding of complex issues in their naturalistic setting. The methodology was flexible and hence permitted considerable probing of the participants' experiences. Besides, it enabled the researchers to have a closer relationship with the participants (Harwati, 2019). The multiple interviews and discussions with the participants led to a very close rapport building which facilitated information collection. There are many benefits of using the case study methodology which is evident in this research. However, it does not mean that it is without limitations, as was evident in our study. Many lessons were also learned in this research when it was reflected in how the research was conducted and there was a crucial problem also with this method. Focus group discussion/interview had produced irrelevant information to the study. When being engaged in in-depth interviews, we cannot only listen to just what is wanted as the specific information. Listening to all the background stories as well cannot be avoided. At this stage, the researchers opted for explorative interviews which helped to find the relevant dimensions to ask for and minimize irrelevant information that did not fit with study hypotheses. For this, we first did rapport building with the participants and then undertook a series of interviews up to four/five times to gather the information we were seeking.

The authors attempted to demonstrate creating a mutual understanding of practice and rigor by referring to the range of case studies documented in the published literature, as well as ongoing discussions concerning trustworthiness and the use of case studies in qualitative research practice. To avoid and/or minimize the credibility issues, researchers were guided by only foundational case study methodologists like Merriam and Stake and in some stages critically with Yin during the case study design and process. Another lesson that was learned was that since case study research is the principal qualitative methodological approach, it made a valued contribution to the field in qualitative inquiry, that was considered. Rigor was maintained by the descriptions of methodological stances. Sufficient details were provided so that the readers could make sense of the study design. It also reveals a rationale for key methodological decisions. An adequate description of information collection, the context of the study, and the final report. A detailed explanation of how the initiation of this research, the process of participant selection, the procedures followed for information collection, the manner of the study analysis until the completion of the final draft was described lucidly. Additionally, a detailed portrayal of the case study sites and interview extracts were cited when reporting the exploration results. This helped the researchers to justify the credibility as well.

Descriptions related to what aspect of the case was considered with example or outlier were adequately justified. Otherwise, the methodology can have loopholes. Importantly, the researchers were equally sensitive and alert to the context and people involved in the study, their verbal statements, and non-verbal signs. Careful attention needed to be given about the gestures while asking for any information with the participation. Even their silence during their interviews could be responses for findings. Therefore, the notes of such instances need to be taken. The notes of these gestures were therefore the information for analysis. For both the multicase project and the case studies, the selection of issues/ questions is crucial (Stake, 2006). The study was guided by the guiding themes during interviews with the participants. In this process, there were instances when critical topics were inadvertently missed for which multiple interviews with the participants 
had to be taken. As a result, case studies as a method became useful in researching inclusion since they may also evaluate phenomena in context (i.e. bound) by utilizing numerous information sources and gathering methods (as cited Baxter \& Jack, 2008).

In the case study research, the researchers know that they are external to and not in control of their case study participants and case study sites (Potter et al., 2010). This was experienced with the participants when the timings scheduled for interviews needed to be adjusted to the exams and classes of the students. This caused a flow of information on the guiding themes on which the interview was based. A change in approach was required with the additional interviews than it was planned. Another lesson that learned was the research methodology much guided by a nonpositivist approach notably following the Stake (2006) and Merriam (2009). There was no strict conceptual framework that was structured. This aided in the research being more exploratory in nature and not being confined in a rigid framework which allowed for flexibility in information collection as well. Another point to be kept in mind while undertaking a case study method is that it takes a long time for the researchers to extract information from the participants and hence a longer time in analyzing information alongside (Gaile, 2018). The researchers had to undertake interviews several times to dig for in-depth information. Interviews were conducted with secondary participants as well to get more insights on the issue.

This shows that some challenges within the case study methodology can inevitably affect the conduct of the research. These challenges can be taken as the lesson learns, which can be improved in the forthcoming research by changing the approach in research design. In handling the challenges, the researchers can make changes in the process, and consequently, find solutions to handle these challenges. In some cases, the researchers can capitalize on opportunities that arise or accept a difficult situation and move on. One can reflect on the complicated experience, analyze, and generate new ways of understanding complicated processes in the process of a study.

\section{Conclusion}

A case study methodology is useful to capture the complexity of single or multiple cases when it is conducted in its natural setting, although there are some challenges in it. These challenges within the case study can inevitably affect the conduct of the research, for example by rethinking changes while collecting information from interviews. The concern for us as researchers is therefore how to handle these challenges, to either capitalize on opportunities that arise or accept a difficult situation and move on. Since the researchers are external to the case study participants, the flow of information can get affected which severely might limit the available information. A change in research approach can be adopted in such situations. However, despite the challenges, the case study assists us in an in-depth analysis. It also helps to examine cases from historical to present form and bring out the similarities and dissimilarities in the cases unlike in other forms of methods. This method captured the complexity of school and classroom practice in our study.

The study was able to uncover the complex connections amongst diverse layers of environment that were influencing the school experiences of our participants, and provided explanations at different levels (e.g., the teacher, the peers, the classroom, the school, and its context). This method also offered a way in which to conclude the multiple layers that play as enablers or barriers in inclusion. However, the rationale for purposive selection of cases needs to be well justified to make the credibility of the study strong, in addition to collecting the necessary detail for gaining a deep understanding of the dynamics at interplay and reasons embedded in the processes undertaken. The challenges in the process of the case study also allow the researcher to develop and improve their skills as case study researchers. Despite having some advantages and 
limitations using the case study method, it is of utmost importance if the research questions are answered well keeping in mind the limitations that come with it. Therefore, the authors advise both novice and experienced researchers to carefully analyze their research design choices and to view the case study as a methodology that provides a flexible, multi-perspective research approach, particularly, to carry out a study on inclusion in education.

\section{References}

Baskarada, S. (2014). Qualitative case study guidelines. The Qualitative Report, 19(40), 1-18. https://nsuworks.nova.edu/tqr/vol19/iss40/3

Baxter, P., \& Jack, S. (2008). Qualitative case study methodology: Study design and implementation for novice researchers. The Qualitative Report, 13(4), 544-559.

Bhattarai, P.C. and Maharjan, J. (2016). Ethical decision making among women education leaders: a case of Nepal. In racially and ethnically diverse women leading education: A worldview (advances in educational administration, Vol. 25). Emerald Group Publishing Limited, Bingley, pp. 219-233. https://doi.org/10.1108/S1479-366020160000025013

Bronfenbrenner, U. (1979). The ecology of human development: Experiments by nature and design. Harvard University Press.

Central Bureau of Statistics (CBS). (2012). National population census, 2011. Author.

Creswell, J. W. (2007). Qualitative inquiry and research design: Choosing among five approaches. Sage.

Department of Education. (2007). Samahita shikshha suchhana samagari [Material for inclusive education information]. Inclusive Education Section, Department of Education.

Fatima, G., Akhter, M. S., Malik, M., \& Safder, M. (2013). Difficulties encountered by students with visual impairment in inclusive education at higher education level. Journal of Educational Research, 16(1), 62-72.

Gaile, B. (2018). 12 case study method advantages and disadvantages. https://brandongaille.com/12-case-study-method-advantages-and-disadvantages/.

Gerring, J. (2007). Case study research: principle and practices. Cambridge University Press.

Government of Nepal. (2015). The constitution of Nepal. Author.

Haines, D. (2017). Ethical considerations in qualitative case study research recruiting participants with profound intellectual disabilities. Research Ethics, 13(3-4) 219-232.

Hancock. D., \& Algozzine. B. (2006). Doing case study research: A practical guide for beginning researchers. Teachers College Press.

Harrison, H., Birks, M., Franklin, R., \& Mills, J. (2017). Case study research: Foundations and methodological orientations [34 paragraphs]. Forum Qualitative Sozialforschung / Forum: Qualitative Social Research, 18(1), Art. 19. doi:10.17169/fqs-18.1.2655

Harwati, L. N. (2019). Ethnographic and case study approaches philosophical and methodological analysis. International Journal of Education and Cultural Studies, 7(2), 150-155. doi: 10.7575/aiac.ijels.v.7n.2p.150

Lamichhane, K. (2012). Disability and barriers to education: Evidence from Nepal. Scandinavian Journal of Disability Research, 15(4), 311-324. doi:10.1080/15017419.2012.703969

Mathema KB (2007). Crisis in education and future challenges for Nepal. Retrieved from http://himalaya.socanth.cam.ac.uk/collections/journals/ebhr/pdf/EBHR_31_04.pdf

Mcmanis, L.D. (2017). Inclusive education: What it means, proven strategies and a case study. Concordia University, Chicago, USA.

Merriam, S.B. (2009). Qualitative research: A guide to design and implementation revised and expanded from qualitative research and case study applications in education. Jossey- 
Bass.

Ministry of Education, Science and Technology. (2019). National education policy, 2019. Author.

Morris, C. (2014). Seeing sense: the effectiveness of inclusive education for visually impaired students in further education [Doctoral dissertation]. Cardiff University, UK.

Mukhopadhyay, S., Nenty, H. J., \& Abosi, O. (2012). Inclusive education for learners with disabilities in Botswana primary schools. Sage Open, 2(2). doi:10.1177/2158244012451584

Nepal Law Commission. (2017). Act relating to person with disabilities, 2074 (2017). Author. Nepal Law Commission. (2018). Act related to compulsory and free education (2018). Author.

Potter, L. E., von Hellen, L., \& Nielsen, S. (2010, November). The practical challenges of case study research: Lessons from the field. In 5th Conference on Qualitative Research in IT (pp. 29-30). https://www.researchgate.net/publication/50370143

Rashid, Y., Rashid, A., Warraich, M. A., Sabir, S. S., \& Waseem, A. (2019). Case study method: A step-by-step guide for business researchers. International Journal of Qualitative Methods, 18, 1-13. doi:1609406919862424

Regmi, N.P. (2017). Inclusive education in Nepal: From theory to practice [Unpublished doctoral dissertation]. Ludwig-Maximilians-University, Germany.

Stake, R. (2006). Multi case study analysis. Guilford Press.

Thapa, S. B. (2012). Schooling of girls with disability: A phenomenological study of Nepali girls [Unpublished doctoral dissertation]. Kathmandu University School of Education, Dhulikhel, Nepal.

Vygotsky, L. (1998). The collected works of L.S. Vygotsky. In Child psychology. Lenum Press.

Yazan, B. (2015). Three approaches to case study methods in education: Yin, Merriam, and Stake. The Qualitative Report, 20(2), 134-152. https://nsuworks.nova.edu/tqr/vol20/iss2/12

Yin, R.K. (2011). Qualitative research from start to finish. The Guilford Press.

Yin, R.K. (2018). Case study research and application: Design and methods. Sage.

\section{Notes on Contributors}

Prativa Shrestha is a doctoral student in the Department of Development Education at Kathmandu University- Nepal. On the professional front, she is working as a Senior Program Coordinator in Education Unit for UNESCO Kathmandu in providing coordinated policy advice, technical assistance, capacity building, designing of education sector development plans in support of the national educational priorities, such as curriculum reform, teacher policies, ICT in education and monitoring of education progress. Her area of research is on inclusive education with a focus on people with disability.

Prakash C Bhattarai is an Associate Professor and Head- Department of Development Education, Kathmandu University-School of Education, Nepal. He teaches research methods, development theories, project evaluation, and researches in the diverse areas of education and social science. He has been working as the faculty, project evaluator, researcher, and trainer in several international and national organizations. Some of his many experiences in the field of writing emanate from his diverse experiences. He has a keen interest in ethics, anti-corruption, development education, and vocational education. https://orcid.org/0000-0003-4804-7492

Manuscript received August 31, 2021 Final revision received November 23, 2021 Accepted December 26, 2021 